\title{
Editorial
}

\section{Special Issue on Machine Learning for Biomedical Data Analysis}

\author{
Federico Divina *(D) and Francisco Gómez-Vela *(D) \\ Computer Science Division, Pablo de Olavide University, ES-41013 Seville, Spain \\ * Correspondence: fdivina@upo.es (F.D.); fgomez@upo.es (F.G.-V.)
}

Received: 14 October 2019; Accepted: 24 October 2019; Published: 2 November 2019

In our world, increasing amounts of data are produced everyday. These data come from everywhere: sensors used to gather climate information, posts to social media sites, digital pictures and videos, purchase transaction records, and cell phone GPS signals, are just a few examples. These data include big data. This trend also affects biology and medicine, where new techniques, e.g., next generation sequencing, allow the production of more data than ever. Moreover, the data generated may be of different natures: text, images, gene expression, signals, etc. In addition to this, typically such data present a high presence of noise. It follows that there is a clear need to analyze and extract useful information from such data. In this sense, machine learning (ML) techniques provide the ability to analyse these data and extract relevant information from it, or even make predictions about it.

The overall aim of this special issue is to compile the latest research and development, up-to-date issues, and challenges in the field of ML and its applications in bioinformatics and medical applications.

As a result, eight papers were accepted and published in this special issue. Among the different machine learning techniques used, deep learning has emerged as a very popular technique in the field of biomedical applications. This fact is confirmed by the article from Zemouri et al. [1], which proposes a review on the use of deep learning in the biomedical field, showing how the popularity of this technique has grown within this field. In particular, the authors provide an overview of the recent status of deep learning in biomedical applications. More specifically, it appears that there is a growing interest in convolutional neural networks (CNNs). This observation is confirmed if we consider the next three articles.

In fact, in the article from Lih Oh et al. [2], a CNN was applied for the diagnosis of schizophrenia. In particular, electroencephalogram signals were collected from healthy patients and from patients presenting with schizophrenia. The proposed strategy achieves high classification accuracy, showing that it could be used for this kind of diagnosis.

CNNs were also used in [3]. In particular, CNNs were applied for the diagnosis and localization of glaucoma, achieving a high prediction accuracy. Moreover, the authors present a publicly-available prototype web application for computer-aided diagnosis and localization of glaucoma, integrating the most effective predictive model in its back-end.

In Gonella et al. [4], CNNs were also used, together with support vector machines. In this case, these techniques were applied to segment brain metastases on contrast-enhanced T1-weighted magnetic resonance images. The results obtained confirm the performances of these two methods, and also highlight some interesting differences between the two strategies, which depends on the size of the features of the data.

Other ML techniques were also used in the remainder of the articles. For example, in Ben Nouma et al. [5], authors investigate a Kohonen neural network applied to knee osteoarthritis (OA) pathology categorization using knee kinematic data samples and applied the network for osteoarthritis pathology classification. The data used in this article consist of a small sample of high-dimensional 
vectors recording repeated measurements of the temporal variation of each of the three fundamental angles of knee three-dimensional rotation during a walking cycle.

In [6] an objective function and a general synthesis system, called DC2Anet, is proposed. The proposed system employs semi-supervised learning to generate lumbar spine magnetic resonance images from single-sequence computed tomography scans. The method was applied to various CT images, showing its superior performance in comparison with other state of the art methods.

Czmil et al. [7] proposed a method for a non-invasive method of type 1 diabetes detection. The proposed method is based on the physical activity measurement of patients. To this aim, different computational intelligence methods were applied. In particular decision tree forests were able to obtain a high accuracy in the diagnosis of diabetes among children, showing a direct relation between physical activity and this kind of diabetes.

Finally, a comparative study of machine learning techniques for the prediction of kyphosis disease is proposed in [8]. In particular, random forests, support vector machine, and neural networks were compared to a baseline method. Results confirmed that ML methods outperformed the baseline method, and that, in particular, neural networks achieved the best results in this case study.

Funding: This research was funded by Spanish Ministry of Economic and Competitiveness and the European Regional Development Fund, grant number TIN2015-64776-C3-2-R (MINECO/FEDER)

Acknowledgments: We would like to thanks to all the authors and peer reviewers for their valuable contributions to this special issue. This issue would not be possible without their valuable and professional work. In addition, we would also like to take the opportunity to show our gratitude to the Applied Science editorial team for their work, for which this special issue has been a success.

Conflicts of Interest: The authors declare no conflict of interest.

\section{References}

1. Zemouri, R.; Zerhouni, N.; Racoceanu, D. Deep Learning in the Biomedical Applications: Recent and Future Status. Appl. Sci. 2019, 9, 1526. [CrossRef]

2. Oh, S.L.; Vicnesh, J.; Ciaccio, E.J.; Yuvaraj, R.; Acharya, U.R. Deep Convolutional Neural Network Model for Automated Diagnosis of Schizophrenia Using EEG Signals. Appl. Sci. 2019, 9, 2870. [CrossRef]

3. Kim, M.; Han, J.C.; Hyun, S.H.; Janssens, O.; Van Hoecke, S.; Kee, C.; De Neve, W. Medinoid: Computer-Aided Diagnosis and Localization of Glaucoma Using Deep Learning. Appl. Sci. 2019, 9, 3064. [CrossRef]

4. Gonella, G.; Binaghi, E.; Nocera, P.; Mordacchini, C. Investigating the Behaviour of Machine Learning Techniques to Segment Brain Metastases in Radiation Therapy Planning. Appl. Sci. 2019, 9, 3335. [CrossRef]

5. Ben Nouma, B.; Mitiche, A.; Mezghani, N. A Sample-Encoding Generalization of the Kohonen Associative Memory and Application to Knee Kinematic Data Representation and Pathology Classification. Appl. Sci. 2019, 9, 1741. [CrossRef]

6. Jin, C.B.; Kim, H.; Liu, M.; Han, I.H.; Lee, J.I.; Lee, J.H.; Joo, S.; Park, E.; Ahn, Y.S.; Cui, X. DC2Anet: Generating Lumbar Spine MR Images from CT Scan Data Based on Semi-Supervised Learning. Appl. Sci. 2019, 9, 2521. [CrossRef]

7. Czmil, A.; Czmil, S.; Mazur, D. A Method to Detect Type 1 Diabetes Based on Physical Activity Measurements Using a Mobile Device. Appl. Sci. 2019, 9, 2555. [CrossRef]

8. Dankwa, S.; Zheng, W. Special Issue on Using Machine Learning Algorithms in the Prediction of Kyphosis Disease: A Comparative Study. Appl. Sci. 2019, 9, 3322. [CrossRef]

(C) 2019 by the authors. Licensee MDPI, Basel, Switzerland. This article is an open access article distributed under the terms and conditions of the Creative Commons Attribution (CC BY) license (http:/ / creativecommons.org/licenses/by/4.0/). 